\title{
Las Podocarpáceas de los bosques montanos del noroccidente peruano
}

\author{
Podocarpaceae of the montane forests from northwestern Peru
}

\author{
Edgar E. Vicuña-Miñano*
}

Publicación: 08/12/2005

\section{Resumen}

Los bosques montanos del noroccidente peruano son formaciones vegetales que presentan una alta diversidad y endemismos. Lamentablemente, en muchos lugares están sujetos a procesos acelerados de deterioro por la deforestación y colonización, aspecto que los convierte en uno de los ecosistemas más amenazados por el hombre. Lo que ahora queda de estos bosques, alguna vez extensos, son sólo fragmentos. Los bosques montanos relictos son ecosistemas ubicados por encima de los $1000 \mathrm{~m}$ de altitud, en la Vertiente Occidental de los Andes. Es en estos bosques, donde se pueden encontrar especies de coníferas nativas de nuestro país, pertenecientes a la familia Podocarpaceae. Estas especies juegan un rol de suma importancia en la dinámica de estos ecosistemas tan frágiles; no obstante, debido a la calidad de sus maderas, están siendo extraídas de manera alarmante. En el noroccidente peruano existen zonas de bosques homogéneos de Podocarpáceas tal es el caso del bosque de Cachil en la provincia de Contumazá, bosque de TongodQuellahorco en la provincia de San Miguel (en los cuales predomina la especie Podocarpus oleifolius) y los bosques de San Ignacio en Cajamarca, en los que reportamos cinco especies de Podocarpáceas distribuidas en tres géneros: Podocarpus (P. oleifolius, P. macrostachys, $P$ sprucei), Prumnopitys (P. harmsiana) y Nageia (N. rospigliosii), las cuales representan el $50 \%$ del total de especies reportadas para el Perú.

Palabras clave: Podocarpus, Podocarpaceae, Bosques Montanos, Nageia, Noroccidente peruano.

\section{Abstracts}

The montane forests of north-western Peru are plant formations with high biodiversity and numerous endemic taxa. Unfortunately, in many places, they are deteriorated by deforestation and colonization processes, to turning into one of the most threatened ecosystems of the world. At the present, montane forests are reduced to isolated fragments. The relict forests are distributed above $1000 \mathrm{~m}$ on the western slope of the Andes. These forests are habitat for Peruvian native coniferous of the Podocarpaceae family. These species are supporting the fragility of montane forests ecosystems, however they are being cut down in large numbers because of the excellent timber quality. There are some forests largely dominated by Podocarpaceae in north-western Peru, we have the Cachil forest (Cajamarca, Province Contumazá) and Tongod-Quellahorco (Cajamarca, Province San Miguel) which are dominated by Podocarpus oleifolius. The San Ignacio forests (Cajamarca, Province San Ignacio) where we find half the species known from Peru, there are five species from three genera: Podocarpus oleifolius, P. macrostachys, P. sprucei, Prumnopitys harmsiana and Nageia rospigliosii.

Keywords: Podocarpus, Podocarpaceae, Montane Forests, Nageia, North-western Peru.

\section{Introducción}

Los bosques montanos de la vertiente noroccidental de los Andes del Perú son formaciones vegetales importantes por presentar una alta diversidad y endemismos; estos bosques otrora extensos, actualmente, debido a la acción antrópica y los cambios climáticos,

\footnotetext{
*Escuela de Postgrado en Ciencias Biológicas, Mención en Gestión Ambiental, Universidad Nacional de Trujillo

E-mail: edgarbotanico23@hotmail.com
}

son sólo relictos de bosque (Dillon, 1993; Young \& León, 1995).

Al oeste del río Marañón y al sur del Paso de Porculla existen bosques montanos aislados cuya destrucción ha progresado rápidamente durante los últimos 50 años, tal como documentan Sagástegui y Dillon, investigadores dedicados a estudiar la flora de estos importantes ecosistemas, los que mediante colectas y estudios de colecciones de herbarios 
de diferentes bosques ubicados en Cutervo, Canchaque, Cachil, Montesco y Tongod han determinado que éstos aún constituyen algunas de las mayores áreas de bosques montanos relativamente no perturbados, al oeste del río Marañón (Dillon, 1994; Dillon et al., 1995)

Los bosques montanos relictos son ecosistemas distribuidos por encima de los $1000 \mathrm{~m}$ de altitud en la vertiente occidental de los Andes. En estos bosques podemos encontrar especies de coníferas nativas pertenecientes a la familia Podocarpaceae. Estas especies de árboles son importantes en la dinámica de esos ecosistemas tan frágiles. Pese a ello, debido a la calidad de sus maderas, están siendo extraídos de manera alarmante. Las Podocarpaceas son las únicas coníferas nativas del Perú y las que adquieren más importancia al formar parte de los bosques nublados peruanos que se ubican entre los $1200 \mathrm{y}$ 3600 m (Brako \& Zarucchi, 1993; Zevallos, 1998; Vicuña \& Mostacero, 2003).

La poca información que se tiene acerca del número real de estas especies en esta zona y en general en el Perú y el dificultoso proceso de identificación de los géneros en campo, han llevado a la elaboración del presente artículo que pretende ser de utilidad en los futuros inventarios, planes de manejo y conservación de estas áreas, ya que, proporciona información sobre las especies de Podocarpáceas que se pueden encontrar, lugares en que han sido reportadas y una clave para su identificación. Asimismo, se resalta la importancia de los bosques montanos relictos del norte del Perú, como ecosistemas a ser considerados como prioritarios en su conservación.

\section{Material y métodos}

El presente trabajo consistió en un estudio taxonómico basado en colecciones de Podocarpáceas de los bosques montanos de San Ignacio (Dpto. Cajamarca, Prov. San Ignacio) en las siguientes localidades: Selva Andina, Cerro Bolo, Picorana, Buenos Aires, Nuevo Mundo y La Bermeja (Fig. 1). Además se revisaron las colecciones de esta fa- milia realizadas en el norte del Perú, principalmente en los bosques montanos relictos y registradas en los siguientes herbarios: Herbarium Truxillense de la Universidad Nacional de Trujillo (HUT), Herbario de la Universidad Particular Antenor Orrego de Trujillo (HAO), Herbario del Museo de Historia Natural de la Universidad Nacional Mayor de San Marcos de Lima (USM), Herbario de la Facultad de Ciencias Forestales de la Universidad Nacional Agraria La Molina de Lima (MOL).

\section{Las localidades de estudio}

Selva Andina (distrito de San José de Lourdes). Se ubica en la margen izquierda del río Chinchipe y está situada entre los 1600 y $2100 \mathrm{~m}$ altitud (050 40' 25' S; 78 54' 29' W). Pertenece a la zona de vida bosque húmedo Montano Bajo Tropical (bh-MBT), con suelo arcilloso rojizo o rojo amarillento con una delgada capa de humus. En esta localidad el impacto humano es muy fuerte. Los campesinos talan y queman los bosques que están situados a lo largo de las cuencas o aquéllos ricos en humus, con escasa pendiente, con la finalidad de establecer monocultivos como el café o pasturas «invernas» para la crianza de ganado vacuno; por este motivo, el bosque cercano al caserío prácticamente ha desaparecido y sólo queda un bosque fragmentado en las zonas adyacentes. En las excursiones de campo pudimos observar tres estratos: herbáceo, arbustivo y arbóreo (Fig. 2).

Cerro Bolo y Picorana (distrito de San José de Lourdes): Cerro Bolo (4 58' 54' S; 78 53' 39 " W) y Picorana (45 9' 10" S; 78 53' 00", W) presentan bosque pluvial Montano Tropical (bp-MT). A partir de los $2100 \mathrm{~m}$ altitud, las colinas son cada vez más altas y abruptas, la capa de suelo es más delgada y las raíces de los árboles forman un piso acolchonado con muchos agujeros que hacen difícil la subida. Entre los 2300 y $2400 \mathrm{~m}$ altitud se extienden las laderas escarpadas que nos permiten ascender al bajo Picorana o también conocido en los alrededores como cerro «Bolo» o cerro «La Yegua». En la cima del cerro «Bolo» se 


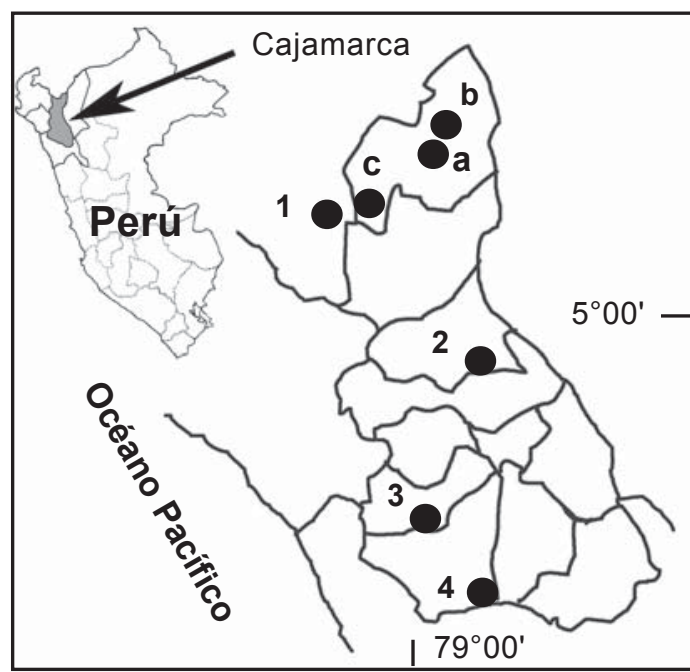

Figura 1. Distribución de las Podocarpáceas en los bosques montanos del norte del Perú. (1) Canchaque, (2) Cutervo, (3) Tongod, (4) Cachil, (a, b, c) San Ignacio.

puede observar un bosque achaparrado; a $2250 \mathrm{~m}$, a través de un camino escabroso, se cruza una cordillera de fuertes pendientes y se llega a la cima del Picorana, que es la montaña más alta del área situada a $2850 \mathrm{~m}$ altitud. Desde el cerro «Bolo» al Picorana el bosque es enano, en la cima se aprecia un campo semiabierto ligeramente plano; el suelo es de arenisca con afloramiento de rocas.

Buenos Aires y Cerro Parco (distrito de San José de Lourdes): Buenos Aires está situada arriba de los $1800 \mathrm{~m}\left(05^{\circ} 04^{\prime} 07^{\prime \prime}\right.$ S $\mathrm{Sy} 78^{\circ} 32^{\prime} 35^{\prime}$ ' W) y pertenece al tipo bosque húmedo premontano tropical. En la parte baja de esta zona se cultivan café, yuca, plátano y caña, también instalan «invernas» para ganado vacuno y solamente quedan manchas de vegetación primaria en los lugares adyacentes al caserío y a las fincas. El cerro Parco es la zona más alta del área (ca. $2550 \mathrm{~m}$ ), motivo por el cual su bosque primario se encuentra casi inalterado $\left(05^{\circ} 04^{\prime}\right.$ 39" S y 78 53' 02" W). A partir de los $2300 \mathrm{~m}$ se observa el bosque enano perennifolio y en la cima del Parco se halla vegetación arbustiva y bosque achaparrado.

Nuevo Mundo-Piragua (distrito de Huarango): situado a $1550 \mathrm{~m}\left(05^{\circ} 14^{\prime} 93^{\prime}\right.$ 'S y $78^{\circ}$ $38^{\prime} 42^{\prime}$ 'W) representa bosque pluvial Montano Bajo

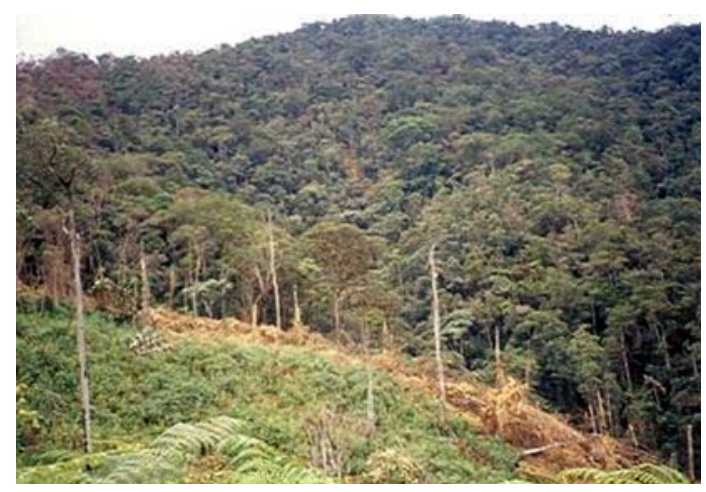

Figura 2. Bosque de Selva Andina en San lgnacio, Cajamarca.

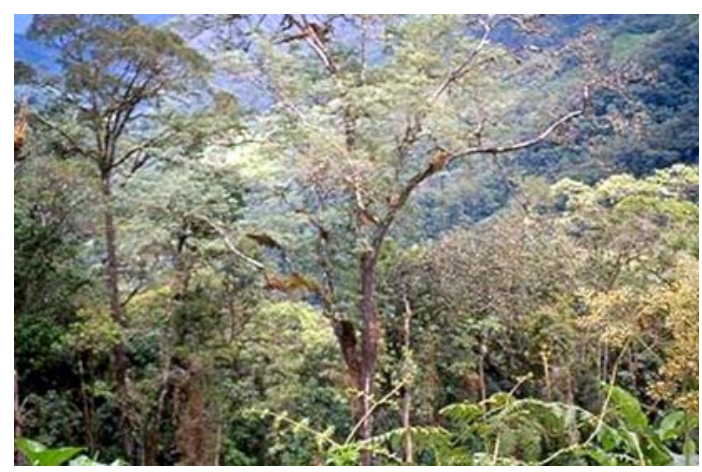

Figura 3. Bosque de La Bermeja en San Ignacio, Cajamarca.

Tropical (bp-MBT). Se trata de una zona de reciente colonización, debido al difícil acceso; por ello aún se pueden observar grandes extensiones de bosque virgen. Debido a su fisiografía tiene muy poco terreno con aptitud agrícola, pese a esto los pobladores establecen cultivos de café, yuca, plátano, con pocos resultados, sólo les alcanza para sus necesidades básicas, la mayor parte de los campesinos establecen pastizales o «invernas».

Bosque de la Bermeja (distrito de Tabaconas): situado arriba de los $1350 \mathrm{~m}$ con el bosque empezando a los $1900 \mathrm{~m}\left(05^{\circ} 23^{\prime} 72^{\prime \prime} \mathrm{S}, 79^{\circ}\right.$ 09' 65' W), se clasifica como bosque húmedo premontano tropical. En esta zona la mayor parte de la población se dedica al cultivo de café, debido a la puesta en práctica del manejo forestal por el proyecto Urubamba, los campesinos también se dedican a trabajar en lo referente a inventario forestal y carpintería. El proyecto Urubamba se dedica a la ex- 
tracción de madera de Podocarpáceas, que en esta localidad abundan; el manejo forestal consiste en extraer y reforestar, lo curioso y que llama la atención es que no reforestan con Podocarpáceas, corriendo el riesgo de poner en grave peligro la existencia de este importante recurso (Fig. 3).

\section{Resultados}

\section{Especies de Podocarpáceas reportadas de la zona}

\section{Nageia rospigliosii (Pilger) Laubenfels}

(Figura 4)

Bol. de Lima, N. ${ }^{\circ} 73$ pp. 57-60. 1991.

= Decussocarpus rospigliosii (Pilger) de Laubenfels, Journ. Arn. Arb. 50: 340. 1969.

= Podocarpus rospigliosii Pilger, Notizbl. Bot. Gard. Berlín 8: 273. 1923

Nombres comunes: «romerillo macho», «romerillo blanco», «diablo fuerte», «alcumano», «utcumanu», «ulcumano».

Reportado para las localidades La Palma, Nuevo Mundo, Buenos Aires, Selva Andina, La Bermeja.

\section{Prumnopitys harmsiana (Pilger) Laubenfels}

Blumea 24: 190. 1978.

\section{(Figura 5)}

= Podocarpus harmsianus Pilger, Pflanzenreich IV. 5/ 18): 68. 1903.

= Podocarpus utilior Pilger ex Fedde, Repert. Nov. Spec.1: 1891905.

= Stachycarpus harmsiana (Pilger) Gaussen, Gymn. Act. Et Foss. 13: 98. 1974.

= Stachycarpus utilior (Pilger) Gaussen, Gymn. Act. Et Foss. 13: 99. 1974.

Nombres comunes: «romerillo hembra», «romerillo colorado», «aymilla», «diablo fuerte», «romerillo rosado», «ulcumano».

Reportado para las localidades de La Palma, Chirinos, Tabaconas, La Coipa, Forestal Andina, Selva Andina, Buenos Aires, La Bermeja y para el departamento de Piura, provincia de Huancabamba, localidad de Manchari.

\section{Podocarpus oleifolius Don}

Pinus 2: 20. 1824.

= P. macrostachyas Parl. in DC. Prod. 16. II. 510. (1868).

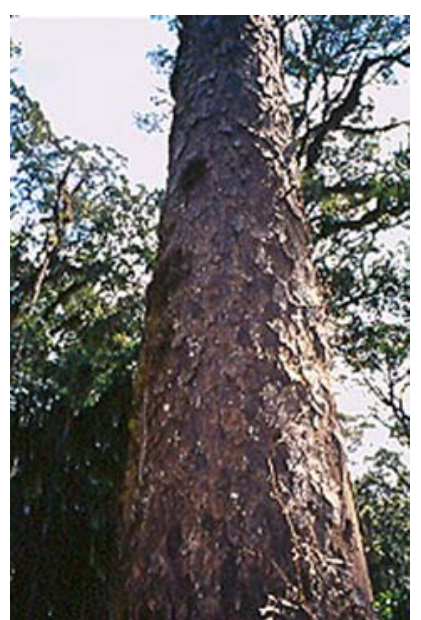

Figura 4. Nageia rospigliosii (Pilger) Laubenfels.

Nombres comunes: «olivo», «romerillo», «saucecillo», «ulcumano de altura»y «ulcumano de puna».

Reportado para el departamento de Cajamarca, provincia deContumazá (bosque de Cachil), provincia de San Miguel de Pallaques (bosque de Tongod, y provincia de San Ignacio, localidades San Andrés de Cutervo, La Palma, Selva Andina, Buenos Aires, La Bermeja y Nuevo Mundo, para el departamento de Lambayeque, provincia de Kañaris (bosques de Kañaris) y provincia de Chiclayo (localidad La Florida), para Piura, provincia de Huancabamba (localidad de La Turmalia, bosques de Huamba).

\section{Podocarpus macrostachys Parlatore ex DC}

Prodr. 16/2: 510. 1868.

= Podocarpus oleifolius var. macrostachyus var. (Parl.) Buchholz \& N.E. Gray, Journ. Arnold Arb. 29: 140. 1948.

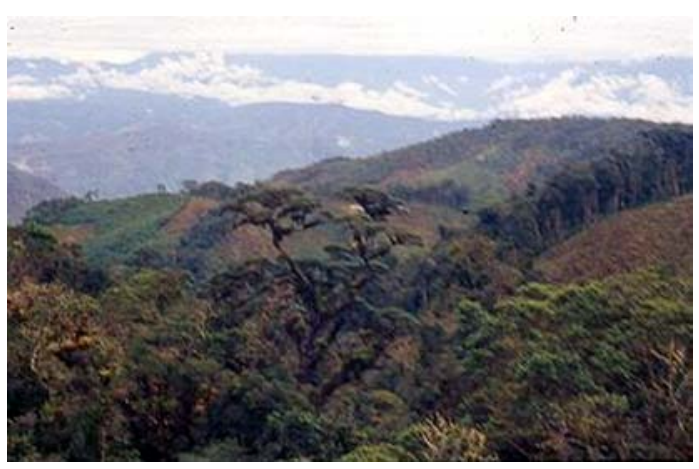

Figura 5. Prumnopitys harmsiana (Pilger) Laubenfels. 
$=$ Podocarpus oleifolius var. trujillensis Buchholz \& Gray. Journ. Arnold Arb. 29: 241. 1948.

Nombres comunes: «olivo», «saucecillo», «pino aparrado», «pinarete».

Reportado para el departamento de Cajamarca, provincia de Contumazá (bosque de Cachil y carretera a Cascas), provincia de Cutervo (1ocalidad Socota), provincia de San Ignacio (localidades Selva Andina, Cerro Picorana), y para el departamento de Lambayeque, provincia de Chiclayo (localidad Torilio).

\section{Podocarpus sprucei Parlatore}

Prodr. (16(2): 510. 1868.

= Podocarpus montanus Willdenow, Sp.PI. IV.4: 857. 1805.

Nombres comunes: «saucecillo», «romerillo».

Reportado solamente para el departamento de Piura, provincia de Huancabamba, cerca Huancabamba (única colección en HUT y HAO).

\section{Clave para los géneros de Podocarpáceae en el norte de Perú}

A. Hojas opuestas, ligeramente decusadas, ovadolanceoladas, 9-16 × 2-4 mm, envés sin tricomas, estróbilos poliníferos en grupos de 3-5 de 6 × 2,5 mm, con escamas poliníferas con acúmen de $1,2 \mathrm{~mm}$.

Nageia

AA. Hojas dispuestas en espiral.

B. Hojas lineares, ligeramente oblanceoladas, de hasta 15 × $2 \mathrm{~mm}$, envés con tricomas ligeramente adpresos estróbilos poliníferos lineales, agrupados en ramitas fértiles, en número de 15-25, de $10 \times 2 \mathrm{~mm}$, escamas poliníferas con acúmen de $0,5 \mathrm{~mm}$.

Prumnopitys

BB. Hoja elíptica-lanceolada, hasta lineal, de 25-85 x 6-12 mm, estróbilos poliníferos solitarios, encorvados, de 18-50 x 4-5 mm, escamas poliníferas con acúmen de $0,8 \mathrm{~mm}$.

Podocarpus

\section{Discusión}

Los bosques montanos relictos por la combinación de diversidad específica y alto número de endemismos se convierten en ecosistemas que representan un elevado potencial económico, paisajístico y ecoturístico, por lo tanto deben ser incluidos dentro de las prioridades nacionales de conservación. Según la CONAM, la vulnerabilidad de los ecosistemas puede medirse relacionando el estado actual de conservación y el tipo, tiempo de acción, escala espacial e intensidad de las amenazas en los siguientes años. Estos factores se pueden correlacionar también con características topográficas, edáficas y climáticas, para dar una idea más precisa de la vulnerabilidad de los ecosistemas. Los bosques montanos del norte del Perú están siendo sujetos a procesos acelerados de deforestación con el fin de ampliar la frontera agrícola y ganadera; asimismo, no existe un plan de manejo forestal para las especies que componen estos bosques (v.g. Podocarpáceas); a ello se suman la topografía compleja y accidentada, así como la condición climática propias de la zona, y que los convierte en uno de los ecosistemas más amenazados por el hombre.

Los bosques montanos, por ser ecosistemas con fuertes pendientes y lluvias, y por estar asentados sobre suelos muy pobres o poco desarrollados, tienen un alto grado de vulnerabilidad, especialmente si están sometidos a una alta tasa de deforestación como es el caso de las vertientes orientales de los Andes. Gentry et al. (1987) señalaban la necesidad de dedicar más atención a los ecosistemas andinos ya que en ellos existe mayor número de especies de hierbas y arbustos, muchas de las cuales son de distribución restringida, a diferencia de los árboles y lianas de los bosques amazónicos cuya distribución es más extendida, de allí la importancia de seguir realizando estudios en los bosques relictos de la parte noroccidental de los Andes. Una evaluación regional reciente, efectuada por el Banco Mundial y el WWF para priorizar las acciones de conservación en las diversas ecorregiones terrestres de Latinoamérica, ha resaltado la importancia de los bosques relictos de las vertientes occidentales andinas como uno de los ecosistemas a priorizar en su conservación. Esto debe ser aprovechado por los investigadores avocados al estudio de estos hábitats para la realización de proyectos que combinen la conservación de estos importantes ecosistemas con el desarrollo del país. 
Producto de la revisión del material de herbario y del material de campo de la provincia de San Ignacio, se puede afirmar que los bosques montanos relictos del noroccidente peruano y los bosques de San Ignacio, son los últimos refugios de los «saucecillos»y «romerillos» especies de la familia Podocarpaceae, que son de suma importancia conservar por ser las únicas coníferas nativas del país y por su rol ecológico como especies dominantes en los bosques relictos, así como por su elevado valor económico. En el noroccidente peruano, las zonas donde podemos encontrar bosques homogéneos de Podocarpáceas es en los bosques relictos de Cachil en la provincia de Contumazá y en el bosque de Tongod-Quellahorco en la provincia de San Miguel en los cuales predomina la especie Podocarpus oleifolius; también, aunque en menor cantidad, en el reciente reporte del bosque montano relicto en Kañaris. Los bosques de San Ignacio en Cajamarca, aunque no son considerados bosques relictos, son de suma importancia por presentar poblaciones importantes de los tres géneros de la familia Podocarpaceae existiendo predominancia de los géneros Nageia y Prumnopitys.

Se reportan cinco especies de Podocarpaceas de los bosques montanos del norte del Perú distribuidas en tres géneros: Podocarpus (P. oleifolius, $P$. macrostacys y $P$. sprucei), Prumnopitys (P. harmsiana) y Nageia (N. rospigliosii), las cuales representan el $50 \%$ de las especies reportadas para el Perú. Cabe resaltar, que la especie Podocarpus oleifolius Don es una de las especies más representativas de los bosques relictos de la vertiente noroccidental de los Andes.

\section{Agradecimientos}

El presente artículo forma parte de la tesis del autor, financiada por la John D. and Catherine T. MacArthur Foundation, bajo el auspicio del Jardín Botánico de Missouri (MO).

\section{Literatura citada}

Brako, L. \& J. Zarucchi. 1993. Catalogue of the Flowering Plants and Gymnosperms of Peru. Monogr. Syst. Bot. Missouri Botanical Garden. EE.UU., vol. 45.1286 p.

CONAM<http://www.conam.gob.pe/chm/ InformeNacional/1erInforme/priorida.htm>.

Laubenfels, D. 1982. «Podocarpaceas». Flora de Venezuela. 11/6/2: 7-41

De Laubenfels, D. 1991. Las Podocarpaceas del Perú. Boletín de Lima 13/73: 57-60.

Dillon, M.O. 1994. Bosques húmedos del Norte del Perú. Arnaldoa 2/1: 29-4-2.

Dillon, M.O., A. Sagástegui, I. Sánchez, S. Llatas \& N. Hensold. 1995. Floristic Inventory and Biogeographic Analysis of Montane Forest Northwestern Peru. Edit. S. Churchill et al., New York Botanical Garden. 251-269 p.

Dillon, MO. 1993. Análisis florístico del Bosque de Monteseco (Cajamarca, Perú) e implicancias para su conservación. Arnaldoa 1/3: 45-63.

Gentry, A. \& C. Dodson. 1987. Contribution of nontres to species richness of a tropical rain forest,. Biotropica 19(2): 149-156.

Hamilton, L. ; J. Juvick \& F. Scaneta, 1993. The Puerto Rico Tropical Cloud Forest Symposium: Introduction and Workshop Síntesis. In tropical montane Cloud Forest Procceding of an International Symposium at San Juan, Puerto Rico. Edit. Hamilton et al.:: 1-16.

Gómez, S. A. 1997. Los Podocarpus de Jaén y San Ignacio (Cajamarca). Lima: Ministerio de Agricultura-PRONAMACHCS

Page, C. N. 1988. New and maintained genera in the conifer families Podocarpaceae and Pinaceae. Notes of the Royal Botanical Garden of Edinburgh 45: 377-395.

Page, C. N. 1990. Pinatae. In: Kramer K. U. \& P. S. Green (eds.), The genera of seed plants I, Pteridophytes and Gymnosperms: 317-346. Springer Verlag, New York.

Vicuña, E \& J. Mostacero, 2003: Notas sobre Podocarpaceas de cuatro Bosques Montanos de la Provincia de San Ignacio- Cajamarca, Perú. Arnaldoa 10(1). 19-44.

Young K. \& B. León. 1995. Peru humid eastern montage forest: An overview of their physical settings, biological diversity 93, human use and settlement, and conservation needs. DIVA, Technical Report n. ${ }^{\circ} 5$.

Zevallos, P. A. 1998. Aportes sobre la Dendrología, Distribución geográfica y status de las Podocarpaceas en el Perú. Tesis para optar el Grado de Magíster. Universidad Agraria La Molina. Lima, Perú. 